\title{
Attentional modulation of oscillatory activity in human visual cortex
}

\author{
Noriko Yamagishi, ${ }^{\mathrm{a}, *}$ Daniel E. Callan, ${ }^{\mathrm{b}}$ Naokazu Goda, ${ }^{\mathrm{a}}$ Stephen J. Anderson, ${ }^{\mathrm{c}}$ \\ Yoshikazu Yoshida, ${ }^{\mathrm{d}}$ and Mitsuo Kawato ${ }^{\mathrm{a}}$ \\ ${ }^{a}$ ATR Computational Neuroscience Laboratories, 2-2-2 Hikaridai, Seika-cho, Soraku-gun, Kyoto 619-0288, Japan \\ ${ }^{\mathrm{b}}$ ATR Human Information Science Laboratories, 2-2-2 Hikaridai, Seika-cho, Soraku-gun, Kyoto 619-0288, Japan \\ ${ }^{\mathrm{c}}$ The Wellcome Trust Laboratory for MEG Studies, Neurosciences Research Institute, School of Life \& Health Sciences, \\ Aston University, Birmingham, B4 7ET, UK \\ ${ }^{\mathrm{d}}$ Technology Research Laboratory, Shimadzu Corp., 3-9 Hikaridai, Seika-cho, Soraku-gun, Kyoto 619-0237, Japan
}

Received 18 February 2003; revised 20 May 2003; accepted 29 May 2003

\begin{abstract}
The effects of attentional modulation on activity within the human visual cortex were investigated using magnetoencephalography. Chromatic sinusoidal stimuli were used to evoke activity from the occipital cortex, with attention directed either toward or away from the stimulus using a bar-orientation judgment task. For five observers, global magnetic field power was plotted as a function of time from stimulus onset. The major peak of each function occurred at about $120 \mathrm{~ms}$ latency and was well modeled by a current dipole near the calcarine sulcus. Independent component analysis (ICA) on the non-averaged data for each observer also revealed one component of calcarine origin, the location of which matched that of the dipolar source determined from the averaged data. For two observers, ICA revealed a second component near the parieto-occipital sulcus. Although no effects of attention were evident using standard averaging procedures, time-varying spectral analyses of single trials revealed that the main effect of attention was to alter the level of oscillatory activity. Most notably, a sustained increase in alpha-band $(7-12 \mathrm{~Hz})$ activity of both calcarine and parieto-occipital origin was evident. In addition, calcarine activity in the range of $13-21 \mathrm{~Hz}$ was enhanced, while calcarine activity in the range of 5-6 Hz was reduced. Our results are consistent with the hypothesis that attentional modulation affects neural processing within the calcarine and parieto-occipital cortex by altering the amplitude of alpha-band activity and other natural brain rhythms.
\end{abstract}

(C) 2003 Elsevier Inc. All rights reserved.

Keywords: Attention; MEG; Rhythmic activity; Alpha rhythm; Vision; Area V1

\section{Introduction}

Spatially directed attention is important for object recognition and motor control and may aid information processing by enhancing neuronal responses to attended stimuli while at the same time suppressing responses to nonattended stimuli (Posner and Petersen, 1990). Although psychophysical studies have emphasized the role attention may play in high-level visual processing (Treisman and Gelade, 1980), physiological studies have shown that attentional

\footnotetext{
* Corresponding author. Fax: +81-774-95-1236.

E-mail address: n.yamagishi@atr.co.jp (N. Yamagishi).
}

modulation of cell responses is possible within early visual cortex (Moran and Desimone, 1985; Luck et al., 1997), including the primary visual area (V1) (Motter, 1993; Roelfsema et al., 1998; Vidyasagar, 1998; Ito and Gilbert, 1999). The usual finding is that cell responses to a given visual pattern are increased when attention is directed toward it, suggesting that attention somehow acts to enhance the saliency of the pattern.

Neuroimaging studies have confirmed that selected and divided attention play an important role in human visual processing, although opinion varies as to whether area V1 is influenced by attention. A number of event-related potential (ERP) and magnetoencephalographic (MEG) studies report 


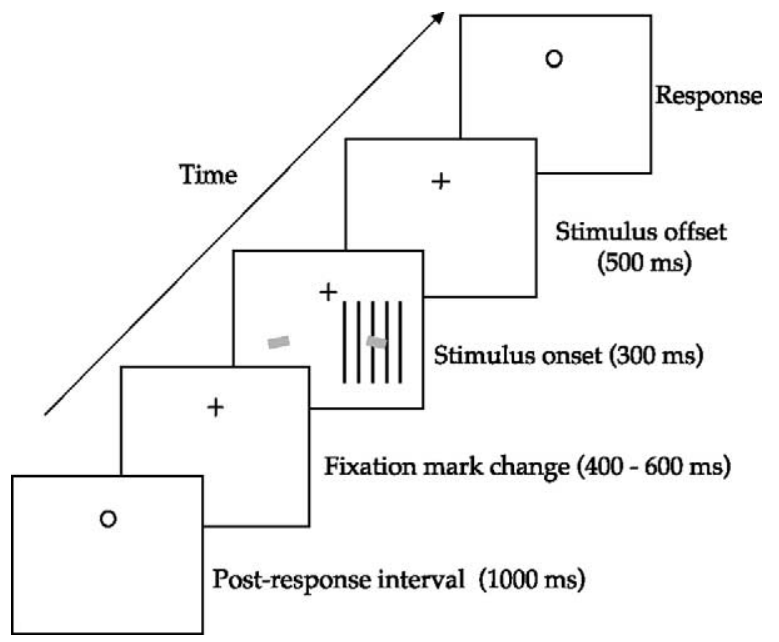

Fig. 1. Illustration of trial sequence. Observers covertly oriented their attention toward the right or left visual field as soon as the fixation mark changed from a circle to a cross. After a variable interval (400-600 ms), the grating stimulus was displayed (duration $300 \mathrm{~ms}$ ) in the lower right visual field. Two small yellow bars were also presented bilaterally in the lower field, the bar on the right being centered on the grating patch. The bars were presented for $100 \mathrm{~ms}$ with a variable onset time of up to $\pm 50 \mathrm{~ms}$ relative to the onset of the grating. The fixation mark reverted back to the circle $500 \mathrm{~ms}$ after the offset of the grating, which was the signal for observers to record their judgment of the orientation of the bar on the side to which they had attended. Following this judgment, for which no response deadline was imposed, the fixation circle remained for a further $1000 \mathrm{~ms}$ before reverting to a cross. An on-screen instruction was presented once at the beginning of each block of trials informing observers which direction to attend.

that activity within V1 is not influenced by attention even though activity within extrastriate visual areas is (Heinze et al., 1994; Clark and Hillyard, 1996; Imada et al., 1996; Mangun et al., 1998; Martinez et al., 1999). In contrast, functional magnetic resonance imaging (fMRI) studies show that attention can enhance visual responses within human V1, as well as extrastriate cortex (Watanabe et al., 1998; Brefczynski and DeYoe, 1999; Gandhi et al., 1999; Martinez et al., 1999, 2001; Somers et al., 1999; Smith et al., 2000).

The discrepant neuroimaging results may reflect the fact that the physiological basis of electromagnetic response measures (ERP, MEG) differs from that of hemodynamic (fMRI) measures (Hamalainen et al., 1993; Logothetis et al., 2001). However, this explanation is weakened by evidence that fMRI (Tootell et al., 1995; Barnes et al., 2001; Singh et al., 2002) and MEG studies (Anderson et al., 1996, 1999; Singh et al., 2002) on early visual areas are in close agree- ment. An alternative explanation is that the attentional modulation of V1 activity observed with fMRI reflects delayed, reentrant feedback from higher visual areas (Vidyasagar, 1998; Martinez et al., 2001). This feedback hypothesis is supported by animal studies (Mehta et al., 2000) and potentially explains why the effects of attention are not reflected in averaged ERP responses (Martinez et al., 2001).

The nature of the attention-related activity in human V1 remains unclear. Attentional processes could lead to a modulation of evoked activity that is phase-locked to the stimulus (ERP) and/or a change in induced oscillatory (rhythmic) activity that is not phase-locked to the stimulus. The latter may take the form of an event-related desynchronization or an event-related synchronization (Pfurtscheller and Lopes da Silva, 1999). Hemodynamic measures cannot distinguish between phase-locked and non-phase-locked activity, whereas electromagnetic response measures can. Both of these hypotheses were examined in this study using MEG. This technique has a spatial resolution approaching that of fMRI for shallow sources (Singh et al., 2002) and allows us to investigate both the locus of attentional modulation and the effects of attention on various natural brain rhythms.

Red/green modulated gratings were chosen as our stimuli because they evoke strong responses from the occipital cortex (Engel et al., 1997; Fylan et al., 1997; Anderson et al., 1999; Anderson, 2002). Covert attention was directed either toward or away from the stimulus using a bar-orientation judgment task. Independent component analysis (ICA) and dipolar source modeling were used to identify the spatial origin of the recorded MEG signals. We were particularly interested in the effects of attention on activity within the calcarine and parieto-occipital sulci, the former because of its disputed role in attentional processing and the latter because of its proposed role in modulating attentional demand (Vanni et al., 1997). Because oscillatory activity in humans might have a critical role in object representation and attention (Ray and Cole, 1985; Vanni et al., 1997; Tallon-Baudry and Bertrand, 1999; Fries et al., 2001), scalograms (time-varying spectral analysis) were computed to compare neural activity under both experimental conditions: attention directed toward the stimulus and attention directed away from the stimulus. We tested whether any observed differences of oscillatory activities were phaselocked to the stimulus onset by calculating intertrial phase coherence at a particular frequency, called "intertrial coherence" (Makeig et al., 2001, 2002) or "phase-locking factor" (Tallon-Baudry et al., 1996).

Fig. 2. Global magnetic field power (GFP) plotted as a function of time from stimulus onset (vertical dotted line) for five observers. The GFP was calculated as GFP $(t)=\sqrt{\sum_{i=1}^{N}\left(S_{i}(t)\right)^{2} / N}$, where $S_{i}(t)$ is the observed magnetic signal in channel $i$ at time $t$, and $N$ is the number of channels. The GFP is shown for both experimental conditions: attention directed toward the stimulus (red line) and attention directed away from the stimulus (blue line). The left panels show the GFP for the observed MEG data. The right panels show the reconstructed MEG functions of the independent components representing activity in Brodmann's area 17. The IC number is shown in the upper right-hand corner of each panel. 
Observed MEG

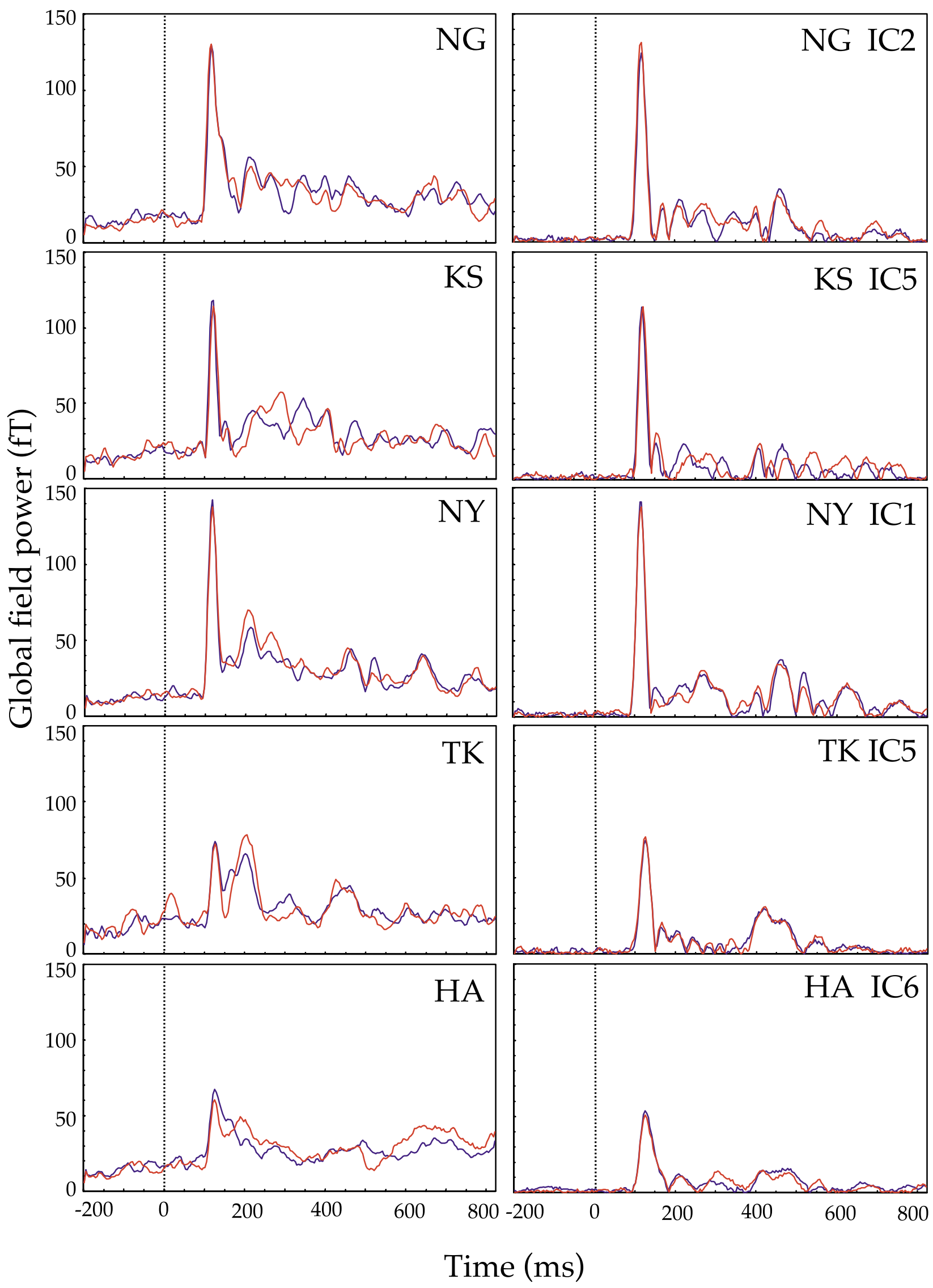

Reconstructed MEG 
(A) Observed MEG
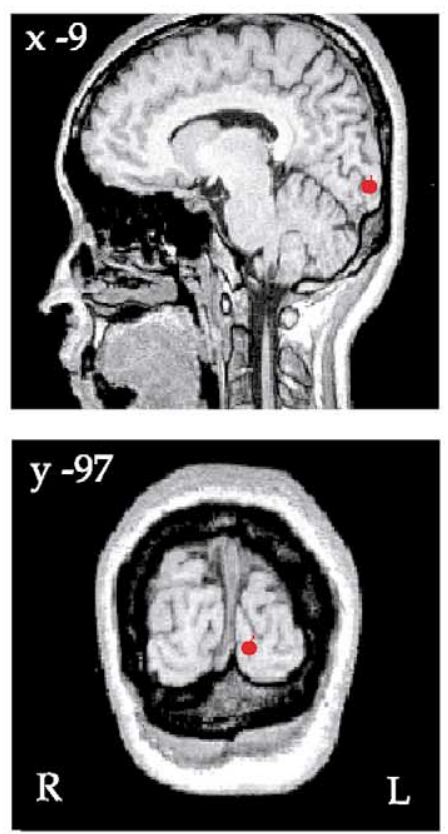

ECD

\section{(B) IC representing calcarine activity}
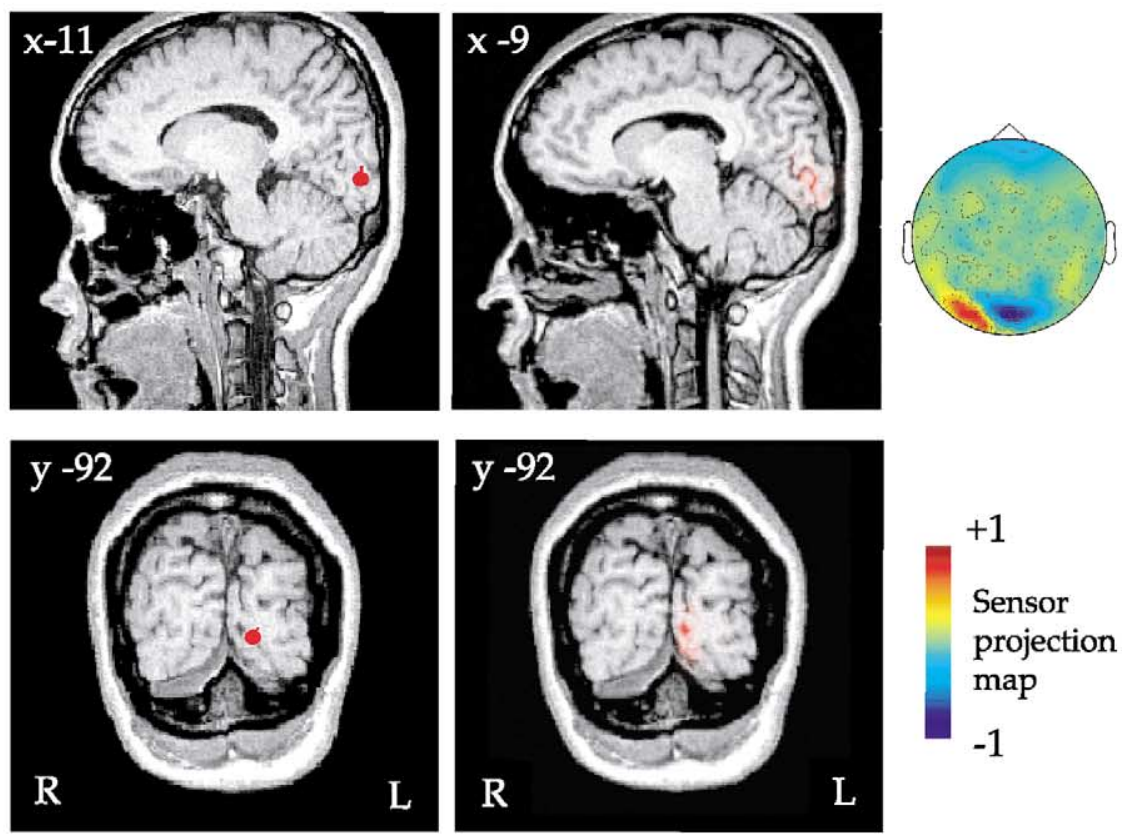

ECD

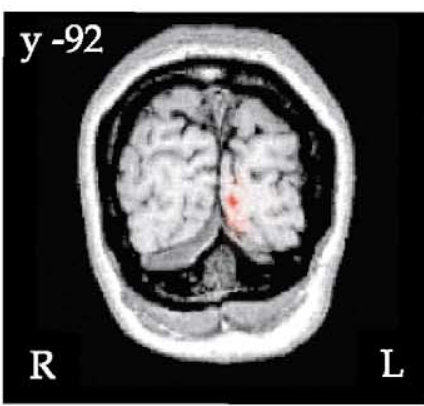

MMM

Fig. 3. Dipole source solutions to the responses evoked using a chromatic grating during the condition whereby attention was directed toward the stimulus, coregistered with sagittal and coronal MR images for observer TK. (A) The single equivalent current dipole (ECD) source solutions (solid red circles) calculated at the latency corresponding to the major peak in the observed MEG data. (B) Source solutions of the independent components (ICs) representing activity in Brodmann's area 17, calculated using both the ECD model and the moving mesh method (MMM, $n=32$ ). The orientation of the single dipole solution is indicated by the orientation of the short red line segment attached to each circle. The appropriate Talairach coordinate for each solution is given in the upper left-hand corner of each plot. The sensor plane projection map $\mathrm{W}^{-1}$ for observer TK is shown on the far right.

\section{Methods}

\section{Subjects}

Five subjects (ages 30-41, three males and two females) with no history of neurological injury were employed. All subjects gave written informed consent for the experimental procedures, approved by the ATR Human Subject Review Committee. All observers had normal or corrected-to-normal visual acuity and normal visual fields and color vision.

\section{Stimuli}

Sinusoidal gratings were generated using a VSG2/3 graphics board (Cambridge Research Systems, UK) and projected (ELP-710 projector, Epson, Japan) from outside a magnetically shielded room (Tokin Corp., Sendai, Japan) onto a semi-translucent screen inside the room. The gratings were modulated in color along the L-M cardinal direction of color space (Krauskopf et al., 1982) around an average white point (CIE coordinates: $x=$ $0.31, y=0.32$, luminance of $\left.80 \mathrm{~cd} / \mathrm{m}^{2}\right)$. Along this color axis, the excitations of the long (L) and middle (M) wavelength-sensitive cones covary. The magnitude of the color modulations was set at $60 \%$ of the maximum available saturation of the display. The gratings were stationary, oriented vertically, had a periodicity of $1 \mathrm{c} / \mathrm{deg}$, subtended $9^{\circ}$ square at a viewing distance of $170 \mathrm{~cm}$, and were rendered isoluminant using the technique of minimum perceptual flicker. Their absolute phase varied randomly from trial to trial. Stimuli were presented in the lower right visual field, displaced $1^{\circ}$ from the principal meridians to minimize activation of multiple regions of cortex and simplify dipolar source analysis (Jeffreys and Axford, 1972). To ensure that central fixation was maintained during stimulus presentation (Fig. 1), simultaneous recording of the electro-oculogram (EOG) was used to detect and discard epochs with eye movement or blink artifacts. Trials were automatically eliminated if the EOG exceeded $\pm 50 \mu \mathrm{v}$. Most trials $(>95 \%)$ for each 
observer were $< \pm 30 \mu \mathrm{v}$ and no systematic eye movement bias occurred for any observer. Viewing was binocular, with the head stabilized using a chin rest.

\section{Procedure}

Evoked magnetic responses to the onset of grating stimuli were recorded from each observer using a 201-channel, whole-head biomagnetic imaging system (Shimadzu Corp., Kyoto, Japan). There were two experimental conditions: attention was directed toward the stimulus under one condition and away from it under another (see below for details). The responses were sampled at $1 \mathrm{kHz}$ for $1024 \mathrm{~ms}$, beginning $200 \mathrm{~ms}$ before stimulus onset.

Observers maintained central fixation throughout each block of trials. Covert attention was directed toward the right or left visual field-depending on initial instructions-as soon as the fixation mark changed from a circle to a cross (Fig. 1). After a variable interval (400-600 $\mathrm{ms}$ ), the grating patch was presented for $300 \mathrm{~ms}$ in the lower right visual field. Two small yellow bars (CIE coordinates: $x=0.33 y=0.36$ size: $0.4^{\circ}$ vertically by $1^{\circ}$ horizontally) were also presented, symmetrically displaced about the vertical meridian with the bar on the right being positioned in the center of the stimulus. During recording, covert attention was directed toward the stimulus by asking each observer to judge the orientation of the right yellow bar. A total of 100 trials were recorded and the resultant signals were band pass filtered at $1-100$ Hz. Under a separate condition attention was directed away from the stimulus by asking subjects to judge the orientation of the left yellow bar. Again, 100 trials were recorded and the signals were band pass filtered at $1-100$ $\mathrm{Hz}$. On each trial, the bars were rotated from horizontal by about $10^{\circ}$ (clockwise or anti-clockwise with equal probability), an angle predetermined using two alternate forced-choice procedures to yield approximately 85\% correct performance for judging their orientation. This level of performance ensures maximum spatial attention in visual tasks of this nature (Lee et al., 1997). To further ensure maximum attention, the yellow bars were presented with a variable onset time of $\pm 50 \mathrm{~ms}$ relative to the onset of the grating. The instruction to attend the right or left yellow bar was presented near the fixation point at the beginning of each condition. The order of conditions was randomized across subjects.

\section{Data analysis}

To determine the location of evoked neural activity, the data were first modeled using a single-equivalent current dipole (ECD) source model. Best-fit solutions were obtained using a least-squares error fit between the observed magnetic signals and those predicted by the model. The solution yielding the best goodness of fit (GF) was determined as

$$
G F=1-\sqrt{\sum_{i=1}^{N}\left(S_{i}-D_{i}\right)^{2} / \sum_{i=1}^{N} S_{i}^{2}},
$$

where $N$ is the number of channels, and $S_{i}$ and $D_{i}$ are the observed and predicted magnetic signals in channel $i$, respectively.

ICA was conducted (Makeig et al., 2001) over the normalized single-sweep data (Callan et al., 2001) to separate sources in the magnetic signals that are spatially fixed and temporally independent (Makeig et al., 1997). In this analysis, the data from each condition (attention directed toward or away from the stimulus) were combined. The raw data for each single sweep of 201 channels was down sampled from 1000 to $250 \mathrm{~Hz}$ and baseline normalized. Independent components (ICs) were determined by using an unsupervised neural network to train a weight matrix that maximizes the joint entropy between the nonlinearly transformed channel data (Makeig et al., 1997). Multiplying the original input data by the rows of the trained weight matrix gives the activation waveform for each IC. The columns of the inverse weight matrix show the relative sensor projection strengths of the respective components (Makeig et al., 1997). ICs were sorted in descending order of their mean projected variance.

In order to determine the IC of calcarine origin, all sensor projection maps (inverse weight matrix $\mathrm{W}^{-1}$ ) were examined. The activation waveforms of the ICs showing large weights in sensor projection maps over occipital regions were projected onto the sensors by multiplication with the inverse weight matrix $\left(\mathrm{W}^{-1}\right)$ and were then subjected to dipole analysis to determine the source location. Scalograms for each of the single-sweep activation waveforms of the calcarine components were computed using Morlet's wavelet analysis (Auger et al., 1996) designed to represent frequencies from 2.5 to 50 $\mathrm{Hz}$ with 32 steps. The half length of the Morlet analyzing wavelet at the coarsest scale was 16 samples. The time dimension of the time frequency representation was evaluated over the 32 points to produce a $32 \times 32$ point scalogram for each single sweep. In addition to the calcarine components, this method of analysis was also used to examine components in the parieto-occipital cortex.

To investigate the consistency of spectral phase across trials in relation to stimulus onset, single-trial data were sorted using a technique that provides a complex value related to the phase distribution of each time-frequency region at a particular frequency and latency window. The modulus of this value is called the phase-locking factor (Tallon-Baudry et al., 1996) or the intertrial coherence (ITC) (Makeig et al., 2002). It ranges from 0 (non-phaselocked) to 1 (strictly phase-locked). 
Table 1

Talairach coordinates for the equivalent current dipole (ECD) solution at the latency of the major peak in the observed MEG global field power functions for both experimental conditions for five observers

\begin{tabular}{|c|c|c|c|c|c|c|c|c|c|c|}
\hline \multirow[t]{2}{*}{ Observer } & \multicolumn{5}{|c|}{ Attention directed away from stimulus } & \multicolumn{5}{|c|}{ Attention directed toward stimulus } \\
\hline & $\mathrm{BA}$ & $x$ & $y$ & $z$ & $\mathrm{GF}(\%)$ & BA & $x$ & $y$ & $z$ & GF (\%) \\
\hline NG & 17 Left & -1 & -94 & 2 & 98 & 17 Left & -1 & -95 & 0 & 98 \\
\hline $\mathrm{KS}$ & 17 Left & -15 & -90 & 5 & 99 & 17 Left & -15 & -93 & 1 & 98 \\
\hline NY & 17 Left & -6 & -92 & 5 & 99 & 17 Left & -6 & -92 & 3 & 99 \\
\hline TK & $17 \mathrm{Left}$ & -13 & -95 & 5 & 98 & 17 Left & -9 & -97 & 2 & 97 \\
\hline HA & 17 Left & -1 & -97 & 2 & 96 & 17 Left & -3 & -97 & 2 & 96 \\
\hline
\end{tabular}

Note. BA, Brodmann area; GF, goodness of fit (see Methods for definition).

\section{Results}

\section{Behavioral performance for bar-orientation judgement task}

Averaged across observers, performance for the barorientation judgment task was $85.8 \%$ correct for the right visual field (grating patch and bar) and $86.2 \%$ correct for the left visual field (bar only). These results conform with reports by observers that the level of task difficulty appeared equal under both conditions.

\section{Effect of attention on global magnetic field power}

For five observers, the evoked magnetic responses to the onset of chromatic gratings were plotted as global magnetic field power (GFP) over time (Fig. 2, left). In these plots, the stimulus appeared at time zero. Responses are shown for two experimental conditions: one where the observer's attention was directed toward the stimulus (red function) and the other away from it (blue function). In general, the maximum field power, noise level, and morphology of the response functions were similar for each condition and varied little between observers. In all cases, a major peak in field power was evident at about $120 \mathrm{~ms}$. Neither the latency nor the maximum power of this initial peak was affected by the attentional status of the observer (latency: $z=0.27, P>$ 0.05 , power: $z=1.75, P>0.05, n=5$, two-tailed Wilcoxon signed-rank test).

For each observer, the peak in GFP near $120 \mathrm{~ms}$ was well modeled by a single equivalent current dipole located on the occipital pole, above the calcarine sulcus and to the left of the interhemispheric midline (contralateral to the stimulus). This can be seen in Fig. 3A (left), which shows the source solutions for one observer (TK) coregistered with sagittal and coronal MR images of this subject's brain. The Talairach coordinates (Talairach and Tournoux, 1988) for each dipole source are reported for each observer in Table 1. Although secondary peaks of lower power were evident for some observers, in general the signal-to-noise ratio at these later times was too small to allow any further analyses to be conducted.

\section{Extraction of calcarine components using ICA}

ICA was conducted over the normalized single-sweep data to extract calcarine components from other cortical activity. For each observer, independent components showing large weights in sensor projection maps over the occipital region were found. These were subjected to dipole source analysis to determine the location of the underlying neural activity. ICs with sources localized to Brodmann's area 17 , as determined by Talairach coordinates, were assumed to reflect striate activity (Table 2). Note that for each observer, only one IC with the dipole source adjacent to the calcarine sulcus in the left (contralateral to the stimulus) hemisphere was found. The position of this source compared well with that of the sources determined from the magnetic field power plots (compare Fig. 3A with B). Using the moving mesh method (MMM) (Kajihara et al., 2000), the locations and volumes of multiple dipoles $(n=32)$ were

Table 2

List of the ICs with sources localized to area 17

\begin{tabular}{lllllll}
\hline Observer & IC number & Brain region & $x$ & $y$ & $z$ & GF $(\%)$ \\
\hline NG & IC2 & Occipital left cun BA 17 & -1 & -95 & 0 & 98 \\
KS & IC5 & Occipital left cun BA 17 & -14 & -94 & 2 & 99 \\
NY & IC1 & Occipital left cun BA 17 & -5 & -92 & 3 & 3.91 \\
TK & IC5 & Occipital left cun BA 17 & -11 & -92 & 3 & 3.16 \\
HA & IC6 & Occipital left cun BA 17 & -5 & -97 & 2 & 97 \\
\hline
\end{tabular}

The Talairach coordinates and brain regions for the equivalent current dipole of the ICs are shown for five observers. Cun, cuneus; BA, Brodmann area; GF, goodness of fit; pvaf, percentage variance accounted for of the projected IC data to the original observed data. 
estimated. The MMM shows that multiple dipoles were located near the calcarine sulcus (Fig. 3B).

For both experimental conditions, the reconstructed power functions of the ICs representing calcarine activity contained a major peak at around $120 \mathrm{~ms}$, mimicking the power functions derived from the averaged evoked MEG responses (compare left and right panels for each observer in Fig. 2). As with the averaged MEG responses, neither the latency nor the power of the major peak in the reconstructed functions was affected by the attentional status of the observer (latency: $z=0.37, P>0.05$, power: $z=0.135, P>$ $0.05, n=5$, two-tailed Wilcoxon signed-rank test).

\section{Effects of attentional modulation on oscillatory activity within the calcarine}

To determine the effects of attentional modulation on oscillatory activity, we computed time-frequency energy plots (scalograms) for each of the single-sweep activation waveforms of the ICs reflecting calcarine activity using Morlet's wavelet analysis (Auger et al., 1996). For each observer, median scalograms representing frequencies from 2.5 to $50 \mathrm{~Hz}$ over the time scale -200 to $824 \mathrm{~ms}$ were created. The stimulus onset time was zero. The pattern of oscillatory activity was similar for each observer. The group-median $(n=5)$ scalograms show that oscillatory activity within the frequency band $3-20 \mathrm{~Hz}$ was evident under both experimental conditions (Fig. 4). At higher frequencies $(>15 \mathrm{~Hz})$, activity was confined to latencies of between 50 and $250 \mathrm{~ms}$. At lower frequencies $(<10 \mathrm{~Hz})$, however, oscillatory activity was evident both before and after the onset of the stimulus.

In order to determine the common neural processes underlying attentional modulation of calcarine activity, every pixel on the scalogram was submitted to statistical analysis ( $n=5$, Wilcoxon signed-rank test). To constrain statistical differences between the two experimental conditions to time-frequency regions with a sufficient degree of energy for reliable statistical evaluation, each pixel was included in the analysis only if its value was greater than one-half standard deviation below the mean value of all pixels for the combined mean of the two conditions (the area below the dark line in Fig. 4C). Fig. 4C shows a significance map ( $P$ $<0.05)$ of the differences between the two experimental conditions: red indicates a relative increase in energy when attention was directed toward the stimulus, while blue indicates a relative increase in energy when attention was directed away from the stimulus. The results show that the magnitude of oscillatory activity of calcarine origin was dependent on the attentional status of the observer. When attention was directed toward the stimulus, three main effects were observed: (a) an increase in energy was evident in the frequency range of 7-10 Hz (lower alpha-band oscillatory activity), beginning some $100 \mathrm{~ms}$ prior to the onset of the stimulus and lasting for its entire duration; (b) enhanced activity within the frequency range of $13-20 \mathrm{~Hz}$ was evident

\section{(A) Attention away from stimulus}

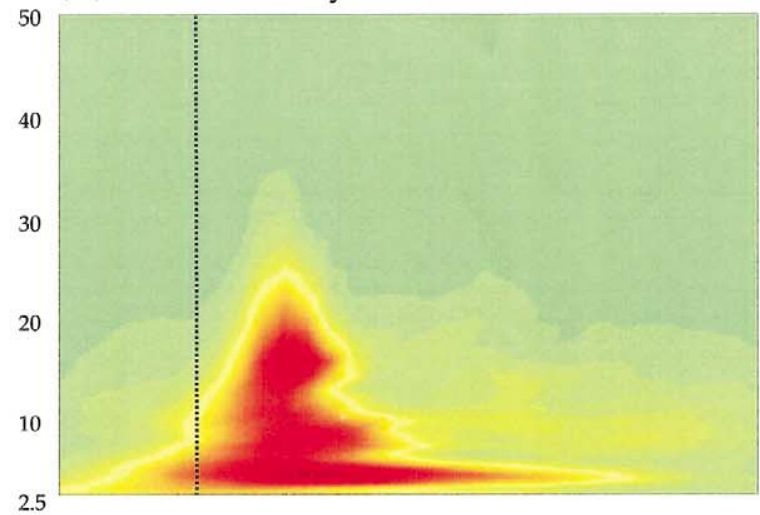

(B) Attention towards stimulus

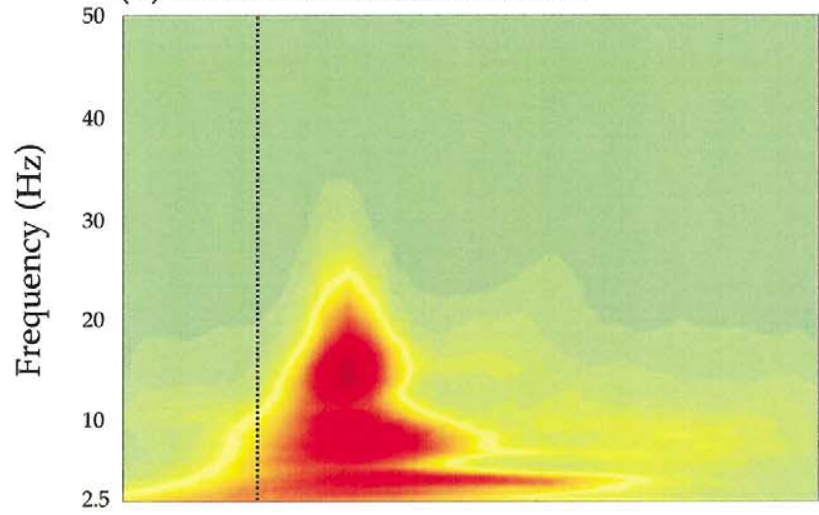

(C) Statistical difference map $(\mathrm{p}<0.05)$

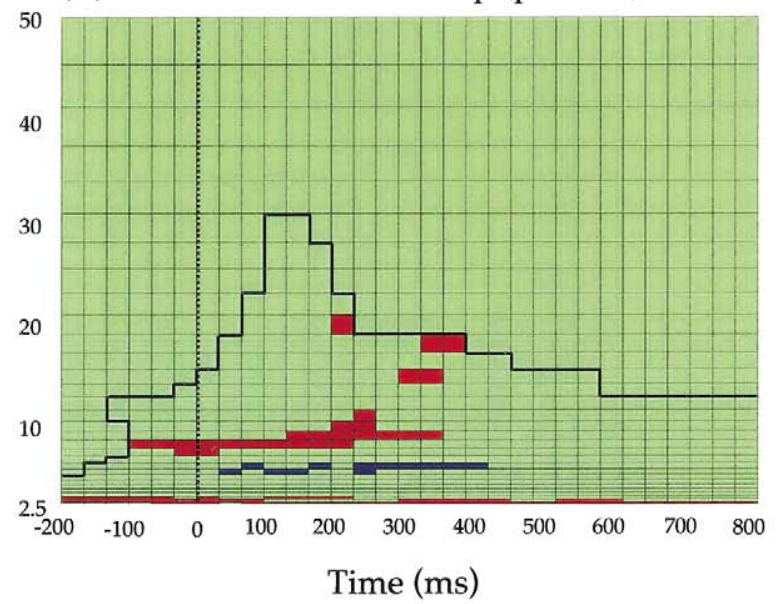

Fig. 4. Group analysis $(n=5)$ showing the effects of attentional modulation on oscillatory activity. Median scalograms for attention directed either away from the stimulus (A) or toward the stimulus (B) (red indicates high-energy spectral density; green indicates low-energy spectral density). (C) Statistical significance map $(P<0.05)$ of the differences between the two experimental conditions. Every pixel that met the criteria (the area below the dark line) on the median scalograms of the IC representing activity in Brodmann's area 17 was statistically evaluated using a two-tailed Wilcoxon signed-rank (nonparametric) test $(z=2.02, P<0.05, n=5)$. The red areas indicate a relative increase in energy when attention was directed toward the stimulus $(P<0.05)$, while the blue areas indicate a relative increase in energy when attention was directed away from the stimulus $(P<0.05)$. In each plot the stimulus onset time is indicated by a vertical dotted line at time zero. 
(A)
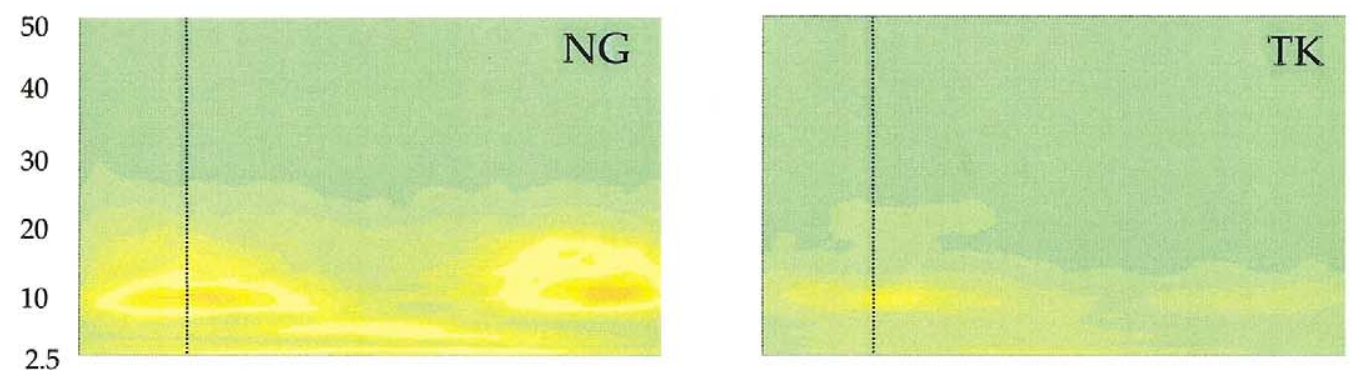

(B)
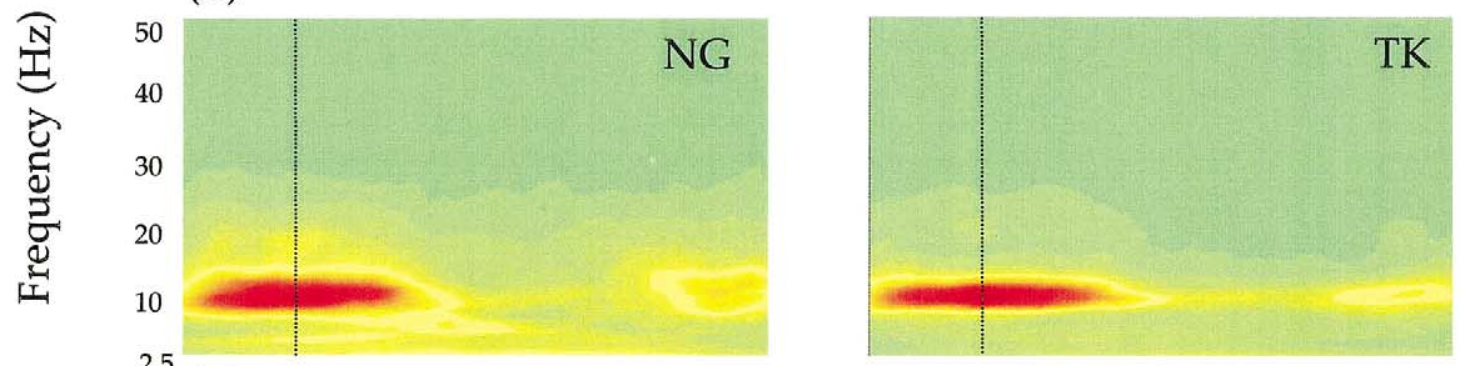

(C)
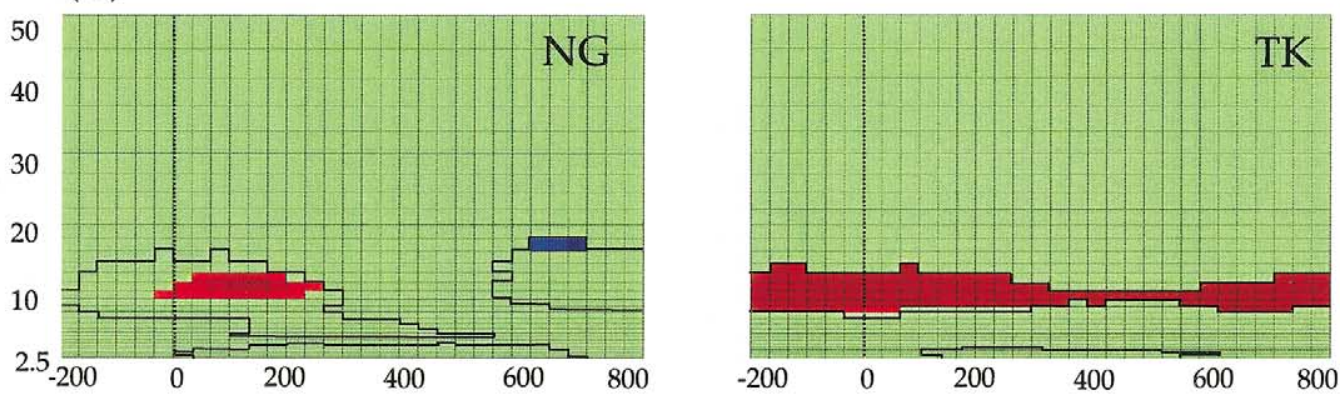

Time (ms)

(D)
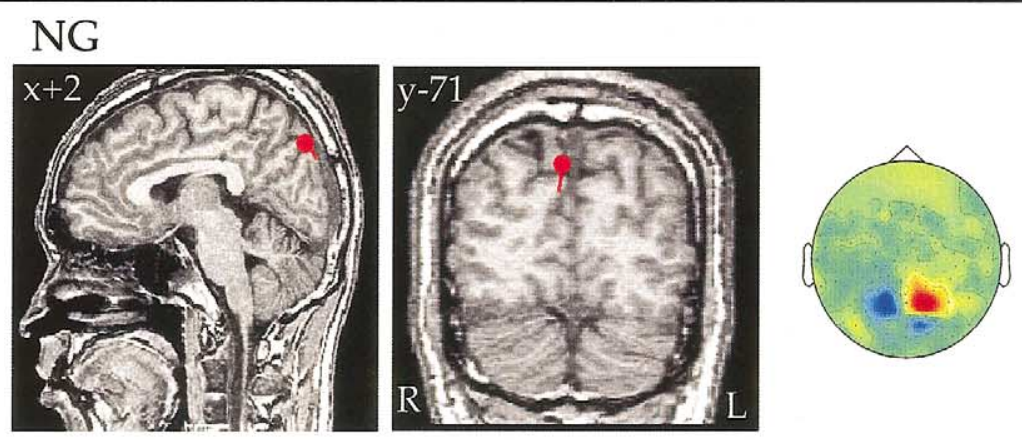

TK
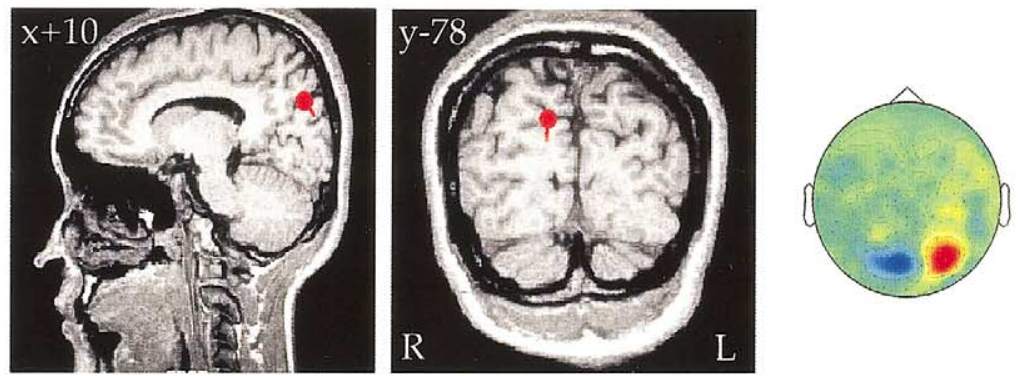
at poststimulus latencies between 200 and $400 \mathrm{~ms}$; and (c) a decrease in energy soon after the onset of the stimulus was evident in the frequency range of 5-6 Hz.

\section{Extraction of parieto-occipital components using ICA}

For two observers (NG and TK), ICs showing large weights in sensor projection maps over the parieto-occipital (PO) region were found. Dipole source analysis confirmed the location of the underlying neural activity to be adjacent to the parieto-occipital sulcus in the right hemisphere (ipsilateral to the stimulus) (see MR images in Fig. 5). Note that this is the opposite hemisphere to that of the identified calcarine components (compare Figs. 3 and 5). For both observers, median scalograms were created using the procedure outlined above to determine the effects of attention on oscillatory activity (Fig. 5A, B). Every pixel on the scalograms of the single sweeps was submitted to statistical analysis ( $n=100$, Wilcoxon signed-rank test) for each observer. Inclusion criteria for the analysis were set as outlined above. The pattern of activity for the PO component was similar for both observers: when attention was directed toward the stimulus, an increase in energy was evident in the frequency range of $10-12 \mathrm{~Hz}$ (upper alpha activity) in the right (ipsilateral) hemisphere (Fig. 5C, $P<$ 0.001 ). The enhanced alpha activity was evident some 200 $\mathrm{ms}$ prior to the onset of the stimulus and lasted for almost its entire duration.

\section{Demonstration of early alpha-band activity in $M E G$ responses}

The spectral analyses reported above show attentional modulation of alpha activity both before and after the onset of the stimulus (Figs. 4C and 5C). However, there remains uncertainty as to whether any or all of the modulation prior to stimulus onset reflects the temporal resolution of the method used: the time resolution at the wavelet scale corresponding to $10 \mathrm{~Hz}$ was $82.6 \mathrm{~ms}$ (half width at half height). Here, we investigate this. No effects of attention were evident in the averaged MEG responses (Fig. 2) and this may be because they were not tightly phase-locked to the stimulus onset. However, attentional effects are evident in the averaged responses from selected sensors if the alpha activity is forced into synchrony (Fig. 6). For three sensors located over the occipital cortex (Fig. 6D), estimates of spectral power and phase were computed for $10 \mathrm{~Hz}$ activity over the time scale -200 to $100 \mathrm{~ms}$ (observer TK). This time scale was chosen because it preceded the major evoked MEG response to the stimulus. The single-sweep traces were phase aligned for the $10 \mathrm{~Hz}$ activity (Fig. 6C) and then averaged (Fig. 6B). Although this procedure negates the principal response at $120 \mathrm{~ms}$ in the MEG data (compare Fig. $6 \mathrm{~A}$ and $\mathrm{B}$ ), it does reveal prominent alpha-band activity under both experimental conditions from at least $150 \mathrm{~ms}$ prior to the stimulus onset to about $200 \mathrm{~ms}$ postonset (Fig. 6B). Little or no alpha was evident after this time, suggesting that the stimulus-evoked activity affected its amplitude and/or phase. For each sensor, the estimated power of the 10 $\mathrm{Hz}$ activity was affected by the attentional status of the observer $(P<0.0001$, unpaired $t$-statistic, two-tailed). Three other observers (NG, KS, HA) showed similar results for sensors located over occipital regions $(P<0.02$, unpaired $t$-statistic, two-tailed).

\section{Consistency of alpha phase across trials for calcarine and parieto-occipital IC components}

To investigate the consistency of alpha $(10 \mathrm{~Hz})$ phase across trials in relation to stimulus onset, the activation waveforms $(n=100)$ of the ICs representing calcarine activity were sorted for two observers (NG and TK) using the technique of Makeig et al. (2001) (Fig. 7). Single-trial waveforms were sorted according to their phase at $10 \mathrm{~Hz}$ using two sorting windows: -200 to $100 \mathrm{~ms}$ (Fig. 7A, prior to the latency of the major peak in GFP) and 0 to $300 \mathrm{~ms}$ (Fig. 7B, including the latency of the major peak in GFP). The results were displayed as color-coded horizontal lines in a rectangular panel for each experimental condition: red indicates positive values and blue indicates negative values. Fig. 7C shows for each observer and for each condition the time course of the intertrial phase coherence at $10 \mathrm{~Hz}$ (blue line): the horizontal red line indicates an ITC significance level of $P=0.01$. Fig. 8 shows the results of this analysis for PO activity.

For the calcarine components (contralateral hemisphere), and for both experimental conditions, ITC rose sharply immediately after stimulus onset (time $=0$ ) and was near perfect $(\mathrm{ITC}=1.0)$ at $125 \mathrm{~ms}($ Fig. $7 \mathrm{C})$, corresponding to the latency of the peak in GFP for observers NG and TK (Fig. 2). When the data were phase-sorted using a window of -200 to $100 \mathrm{~ms}$, a pattern of tilted colored bars with a periodicity of $10 \mathrm{~Hz}$ was evident in the display window during this time (Fig. 7A). This pattern indicates that alpha

Fig. 5. Median scalograms for the ICs (IC1 for both observers) representing activity in the parieto-occipital (PO) cortex for two observers (NG, TK) for attention directed either away from the stimulus (A) or toward the stimulus (B) (red indicates high-energy spectral density; green indicates low-energy spectral density). (C) Statistical significance map $(P<0.001)$ of the differences between the single sweeps of the two experimental conditions for each observer. Every pixel that met the criteria (the area within the dark line) was statistically evaluated using a two-tailed Wilcoxon signed-rank (nonparametric) test $(z>3.3$, $P<0.001, n=100)$. The red areas indicate a relative increase in energy when attention was directed toward the stimulus while the blue areas indicate a relative increase in energy when attention was directed away from the stimulus. (D) Source solutions for the PO components, calculated using the single ECD model. The Talairach coordinates for each solution are: $(2,-71,44)$ for observer NG (goodness of fit $(\mathrm{GF})=98 \%)$, and $(10,-78,33)$ for TK $(\mathrm{GF}=98 \%)$. The sensor plane projection map $\mathrm{W}^{-1}$ for each observer is shown to the right of their MR images. 
phase was not aligned across trials in the prestimulus period or for $100 \mathrm{~ms}$ poststimulus onset. Note that this pattern of tilted bars was absent when a sorting window of 0 to $300 \mathrm{~ms}$ was used (Fig. 7B), indicating that alpha phase was reset (changed) during this time. For both sorting windows, a prominent vertical red bar (width $50 \mathrm{~ms}$ ) was evident at about $125 \mathrm{~ms}$, indicating a common (fixed latency) peak in the activation waveforms (Fig. 7A,B).

In contrast, the ITC for the parieto-occipital components (ipsilateral hemisphere) remained low for both experimental conditions at all times (Fig. 8C). When the data were phase sorted using a window of -200 to $100 \mathrm{~ms}$, a pattern of tilted colored bars with a periodicity of $10 \mathrm{~Hz}$ was evident in the display panel for each condition from -200 to $200 \mathrm{~ms}$. This pattern, which was most pronounced for the condition whereby attention was directed toward the stimulus, indicates that alpha phase was not aligned across trials. Note that this pattern remained unchanged at stimulus onset (time $=0$ ) and was independent of the sorting window used (compare Fig. 8A with B).

\section{Discussion}

\section{Effects of attentional modulation on calcarine activity}

Our results provide evidence that directing attention toward or away from a visual stimulus differentially affects the level of oscillatory activity within the human calcarine cortex. In comparison with the condition whereby attention was directed away from the stimulus, statistically significant $(P<0.05)$ increases and decreases in oscillatory activity within the calcarine were evident when attention was directed toward the stimulus (Fig. 4C). Three main effects were observed, all of which occurred within the hemisphere contralateral to the stimulus (Fig. 3). First, a sustained increase in alpha-band $(7-10 \mathrm{~Hz})$ activity was evident, beginning some $100 \mathrm{~ms}$ prior to the onset of the stimulus. Second, activity in the frequency range of $13-20 \mathrm{~Hz}$ was enhanced between poststimulus latencies of 200 and 400 ms. Finally, attention caused a reduction in slow oscillatory activity $(5-6 \mathrm{~Hz})$ shortly after the onset of the stimulus and persisting for $100 \mathrm{~ms}$ in its absence.

Localization of the ICA sources to the calcarine sulcus with Talairach coordinates corresponding to Brodmann's area 17 (Fig. 3, Table 2) led us to hypothesize that the generator of the oscillations is in area V1, as opposed to immediately adjacent areas V2 and V3. In support of this hypothesis, we note the following: (a) for each observer, only one IC with a dipole source adjacent to the calcarine sulcus was found (Table 2); (b) the position of this source matched that of the source determined from the first significant peak of the stimulus-evoked magnetic responses (compare Fig. 3A with B); and (c) dipole fits to the activity evoked by chromatic sinusoids of the type used in this study vary in a way that is consistent with the cruciform model of V1-namely, dipole orientations show polarity inversions between upper and lower quadrant stimuli (Fylan et al., 1997; Anderson, 2002).

The effects of attention were not evident in either the GFP plots of the neuromagnetic data or the reconstructed power functions of the calcarine activity (Fig. 2), consistent with previous electromagnetic response measures (Clark and Hillyard, 1996). However, attentional affects were evident using time-varying spectral analyses. Therefore, contrary to previous ERP and MEG studies (see Introduction), we conclude that attentional modulation does affect activity within early visual areas, including V1, and may do so by altering the amplitude of various oscillatory rhythms. The attentional modulation effects observed in human V1 using fMRI (Watanabe et al., 1998; Brefczynski and DeYoe, 1999; Gandhi et al., 1999; Martinez et al., 1999, 2001; Somers et al., 1999) may reflect changes in the level of various natural brain rhythms and need not necessarily reflect a feedback process (Martinez et al., 1999, 2001).

The time resolution at the wavelet scale corresponding to $10 \mathrm{~Hz}$ was $82.6 \mathrm{~ms}$ (half width of impulse response function at half height), and as such at least some of the increase in alpha-band activity in the calcarine during the prestimulus period (Fig. 4C) may be artifactual. However, forcing alpha $(10 \mathrm{~Hz})$ activity into synchrony by simply adjusting the phase of the raw data traces, we showed that attentional modulation of alpha was evident in the averaged responses from selected sensors over the occipital cortex (see Fig. 6). Although this procedure does not allow us to comment on the localization of the activity, it seems likely that some of the activity was near the calcarine (Fig. 7) and PO sulci (Fig. 8). The observed alpha increment in the calcarine before stimulus onset may be related to that observed in the $\mathrm{PO}$ area. This view is compatible with physiological studies showing enhanced interareal alpha synchronization in the cat between the primary visual cortex and higher visual areas during periods in which a behaviorally relevant stimulus was expected (Chatila et al., 1992; von Stein et al., 2000).

Enhancement of alpha during the prestimulus period

Fig. 6. (A) Averaged evoked magnetic responses, phase-locked to the stimulus onset, for three sensors located over the occipital cortex (observer TK). Data for attention directed toward the stimulus are shown in red and data for attention directed away from the stimulus are shown in blue. The stimulus onset time is indicated by the vertical dotted line at time zero. (B) Averaged evoked magnetic responses, phase-aligned for $10 \mathrm{~Hz}$ activity, for the same three sensors. The stimulus onset time was between -50 to $50 \mathrm{~ms}$ (shaded area). (C) Histogram showing the estimated phase for $10 \mathrm{~Hz}$ activity before the alignment (shaded bars) and after the alignment (a single black bar). (D) Top view (nose at the top) of the 201 sensor projection map showing the position of the three sensors $(1,2$, and 3) used. An isocontour map of magnetic field strength at $t=125 \mathrm{~ms}$ for the condition whereby attention is directed toward the stimulus is also shown (orange lines indicate a magnetic field emerging from the head). 
(A) Average

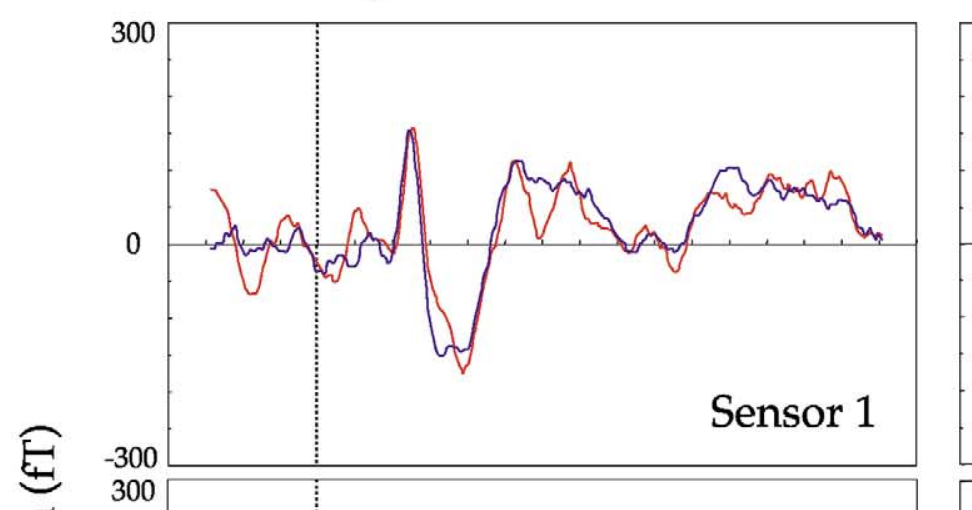

(B) Average (phase aligned)
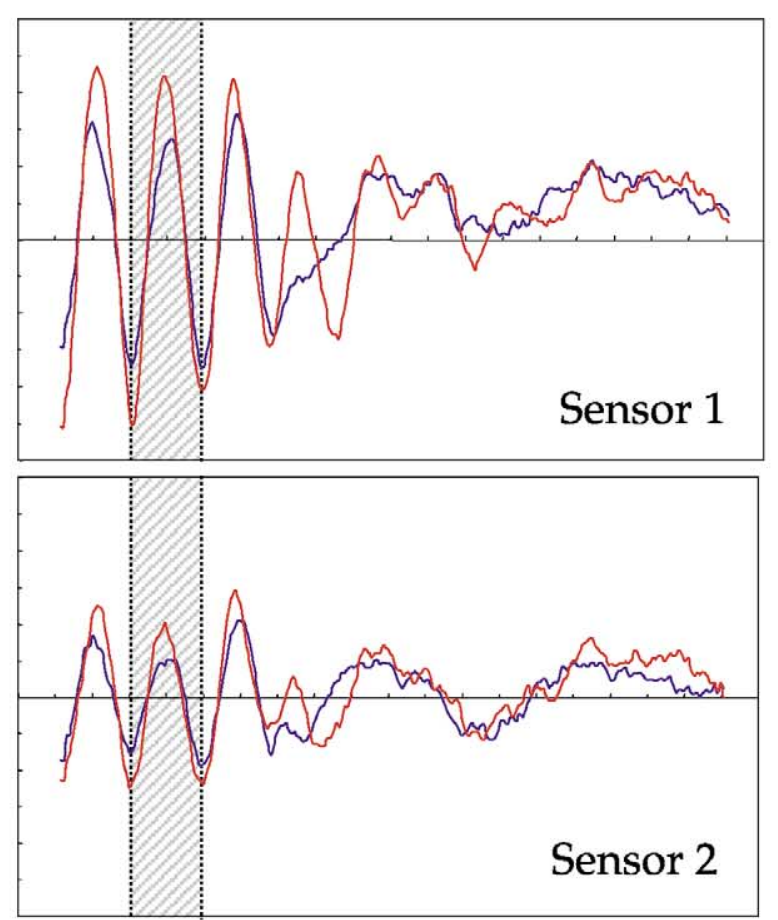

Sensor 2
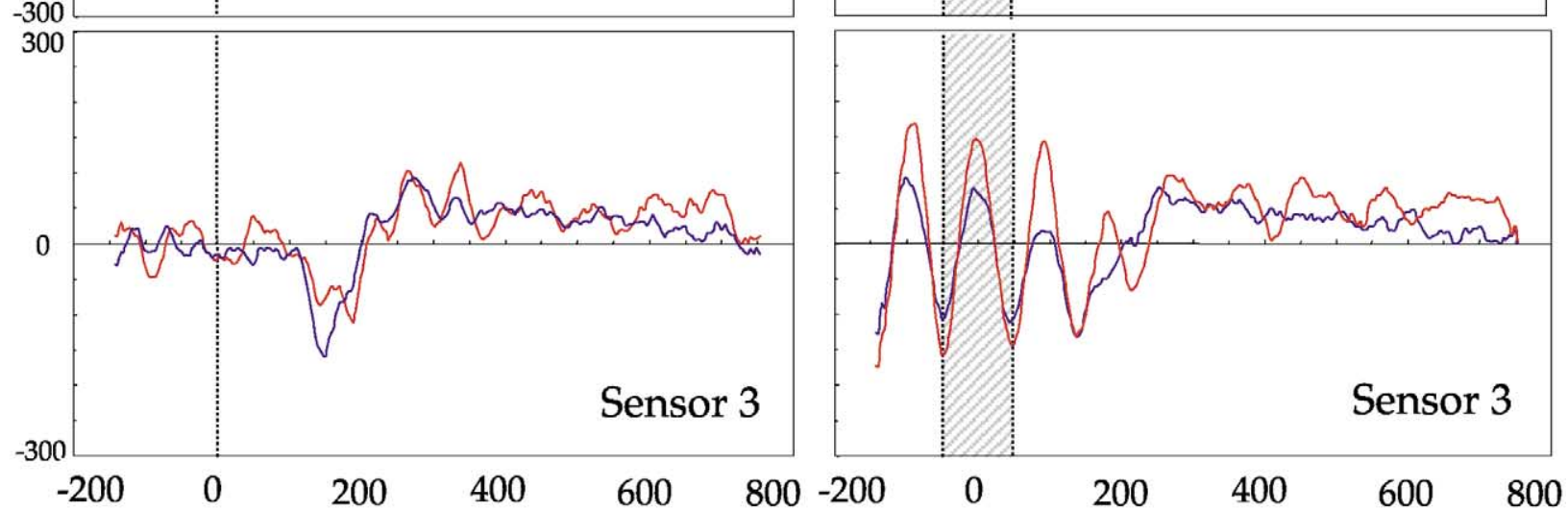

Time (ms)
(D)

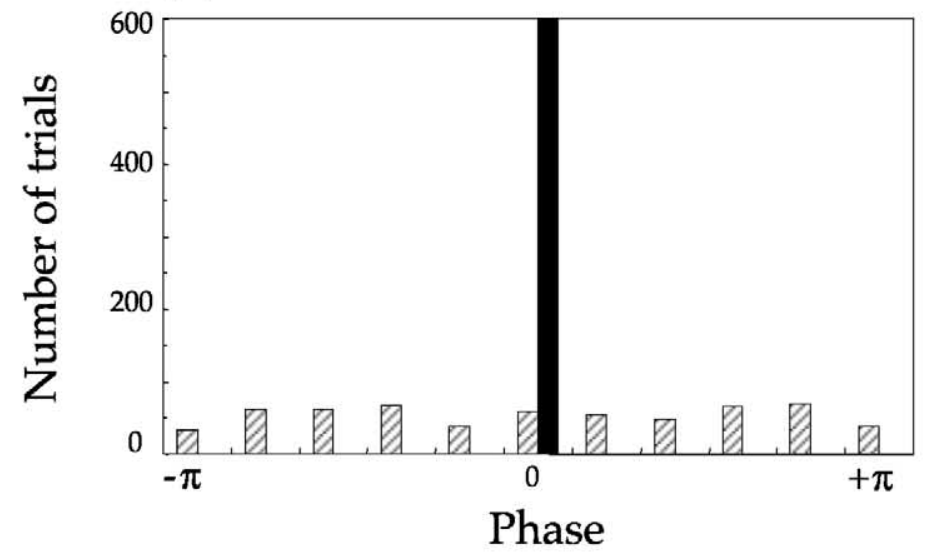

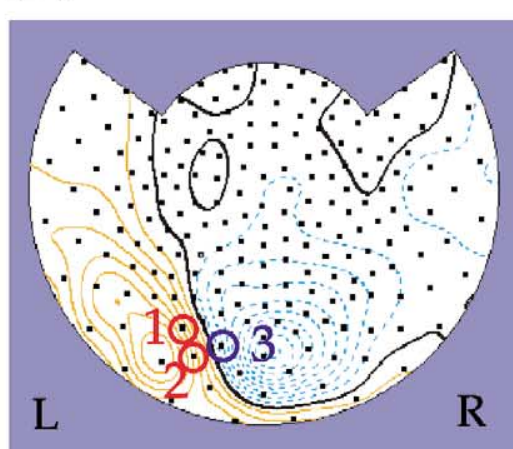


Attention away from stimulus

$$
\text { (A) }
$$

sorting window
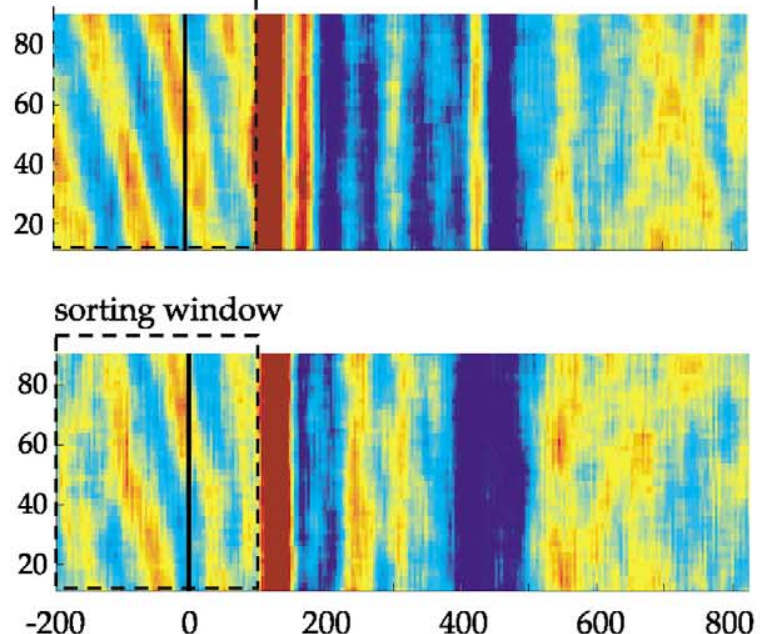

(B)

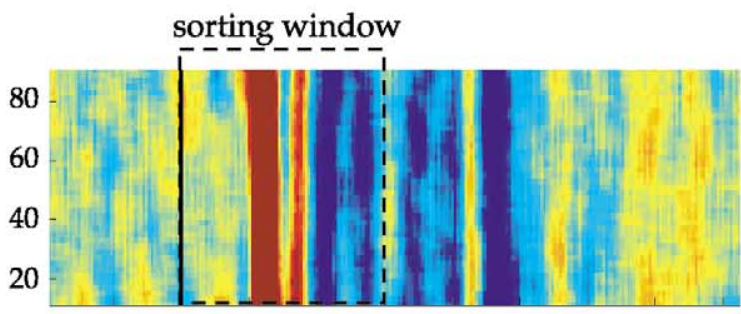

丞

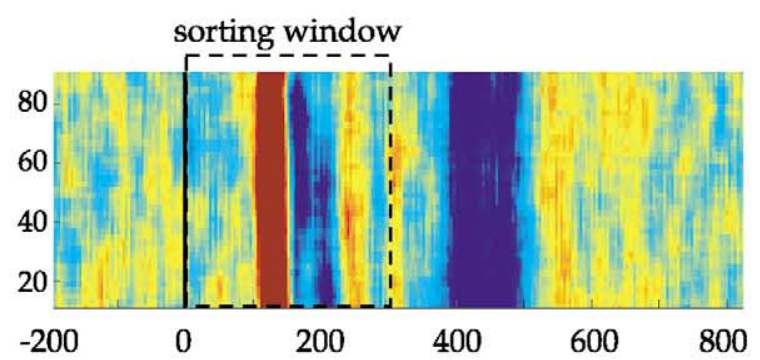

(C)

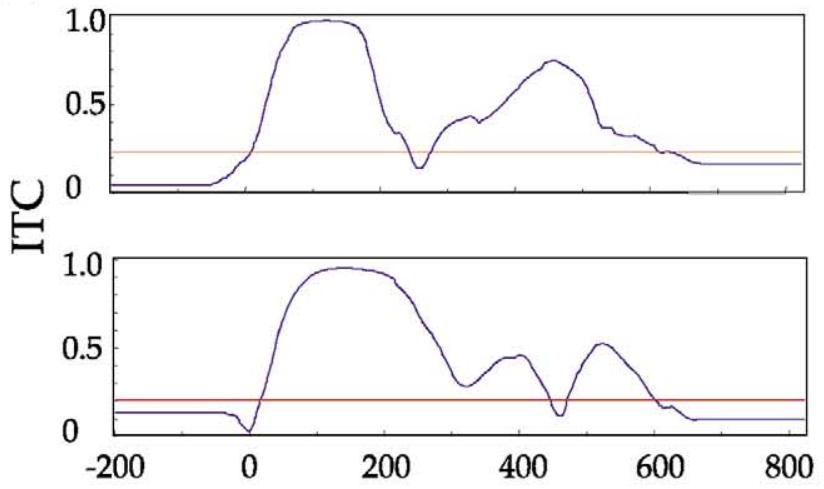

\section{Attention towards stimulus}

sorting window

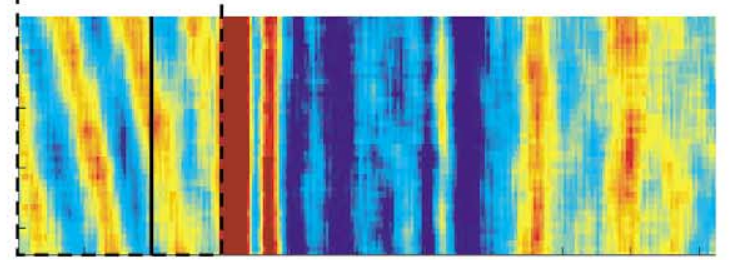

NG

sorting window

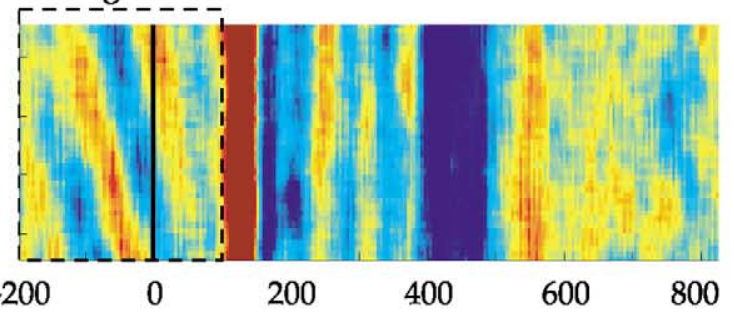

TK

NG

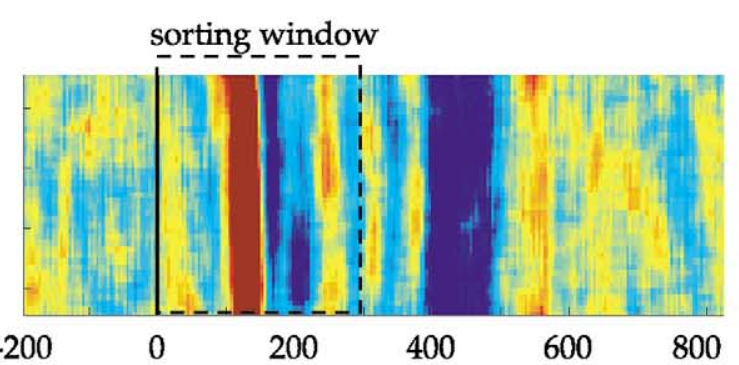

TK

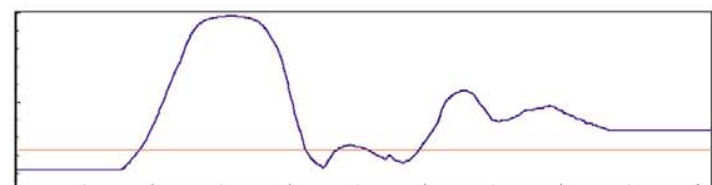

NG

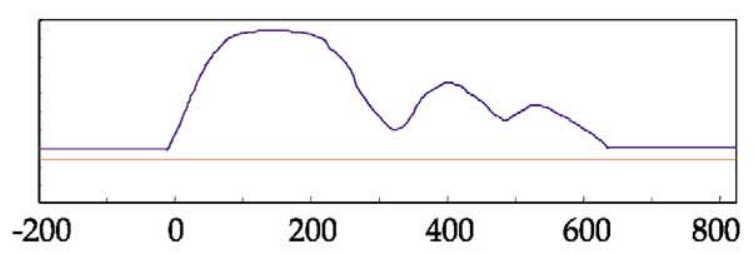

TK

Time (ms) 
(Fig. 4C and Fig. 5C) may reflect self-induced preparatory activity. Enhanced baseline activity in the postcue-prestimulus period in response to attentional shifts has been observed in both single cell studies on monkey (Luck et al., 1997) and fMRI studies on humans (Chawla et al., 1999; Kastner et al., 1999; Ress et al., 2000). For example, it has been reported that hemodynamic responses in motion (V5) and color-sensitive (V4) areas were enhanced during the prestimulus period by selective attention to motion and color stimuli, respectively (Chawla et al., 1999). Changes in baseline activity might form the neurophysiological basis of attentional modulation. This is supported by the observation that increased hemodynamic responses in human extrastriate cortex (Kastner et al., 1999) are qualitatively similar to the increased baseline firing rates of V4 neurons in monkey cortex (Luck et al., 1997). In humans, enhanced hemodynamic responses within striate cortex are evident in some individuals, although they are of lower amplitude and more variable in nature (Kastner et al., 1999). Our neuromagnetic recordings provide evidence to suggest that baseline activity within the striate cortex does increase in response to a directed shift of attention.

\section{Comparison of calcarine and parieto-occipital activity}

When spatial attention was directed toward the stimulus in the right visual field, enhanced alpha-band activity was observed near the calcarine (Fig. 3B) and PO sulci (Fig. 5D). Although the changes in alpha within the calcarine and PO areas followed a similar time course, in most other respects the nature of the changes differed markedly between the two areas. First, enhanced alpha within the calcarine was observed in the left (contralateral) hemisphere, whereas that in the PO area was observed in the right (ipsilateral) hemisphere. Second, the attentional change in alpha was more marked in the PO area than in the calcarine (compare Figs. 4 and 5), although ICA only revealed a PO source in two of five observers. Third, the energy of the signal in the time-frequency plane was spread over a wide range of frequencies in the calcarine (Fig. 4A, B) whereas that in the PO area was confined to about $10 \mathrm{~Hz}$ (Fig. 5A, B). Fourth, in the calcarine, the attentional change in alphaband activity was most pronounced in the frequency range of 7 to $10 \mathrm{~Hz}$ (lower alpha, Fig. 4C), while the change in the $\mathrm{PO}$ area was most evident at 10 to $12 \mathrm{~Hz}$ (higher alpha, Fig. 5C). Finally, analysis of the intertrial phase coherence (ITC) for $10 \mathrm{~Hz}$ activity differed markedly between the two areas. Within the calcarine, ITC rose sharply after stimulus onset and reached a maximum value near 1.0 at a poststimulus latency of about $125 \mathrm{~ms}$ (Fig. 7C), which is indicative of evoked activity or phase resetting (Brandt, 1997; Makeig et al., 2002). In contrast, ITC remained low at all times for the PO area (Fig. 8C). Pictorial representation of the phasesorted data for the PO components revealed a prominent pattern of tilted colored bars, beginning before stimulus onset and persisting for $200 \mathrm{~ms}$ after stimulus onset (Fig. 8), indicating that alpha was not phase-locked to the stimulus onset. Our results provide evidence that the alpha-band activity in the PO area was induced whereas that in the calcarine may reflect both induced and evoked activity.

The differences between calcarine and PO areas reported here lead us to conclude that attentional modulation of alpha within these areas subserves fundamentally different functions. The standard view is that large-amplitude alpha characterizes a deactivated cortical network (Pfurtscheller, 2001). Our results showing increases in alpha during attention tasks appear inconsistent with this view, but they are in agreement with recent studies showing task- and load-dependent increases in alpha activity (Klimesch, 1999; Worden et al., 2000; Jensen et al., 2002). For example, Worden et al. showed that alpha activity increased over the occipital cortex ipsilateral to the direction of attention, which is consistent with our results for two observers showing increased alpha in the right (ipsilateral) PO cortex (see Fig. 5). Increased alpha in the PO area may act to inhibit the processing of information relating to that part of the visual field that is to be ignored. A similar argument was advanced by Worden et al. (2000). However, it is difficult to reconcile an inhibitory role for alpha activity within the calcarine, given that its locus is coincident with that of heightened neural activity associated with visual processing. If we accept the argument that increasing synchrony between neurons may act to increase the synaptic efficiency of a cortical network (Steinmetz et al., 2000), one possibility may be that increased alpha in the calcarine serves to enhance the efficiency of processing information related to the visual stimulus.

The functional relationship between the calcarine and PO areas remains an open question. The various differences between these areas cited above are all consistent with the view that alpha activity within the PO region may modify that within the calcarine, in agreement with the hypothesis that oscillatory processes in the middle frequency range $(4-12 \mathrm{~Hz})$ may involve top-down processes (von Stein et al., 2000).

Fig. 7. Relationship between the alpha $(10 \mathrm{~Hz})$ phase of the activation waveforms $(n=100)$ of the IC representing calcarine activity and stimulus onset (vertical black line at time zero) for two experimental conditions (attention towards/away from stimulus) and for two observers (NG, TK). Single-trial activation waveforms were sorted according to their phase at $10 \mathrm{~Hz}$ using a sorting window of either (A) -200 to $100 \mathrm{~ms}$ or (B) 0 to $300 \mathrm{~ms}$ and displayed as color-coded horizontal lines in a rectangular panel ( + ve values are shown as red and -ve values as blue). The sorting windows are depicted as a dashed square. The sorted trials were then smoothed using a 20-trial moving window. (C) Time course of the intertrial coherence (ITC) at $10 \mathrm{~Hz}$, measuring the degree of phase consistency across trials or phase locking the horizontal red line indicates an ITC significance level of $P=0.01$. 


\section{Attention away from stimulus}

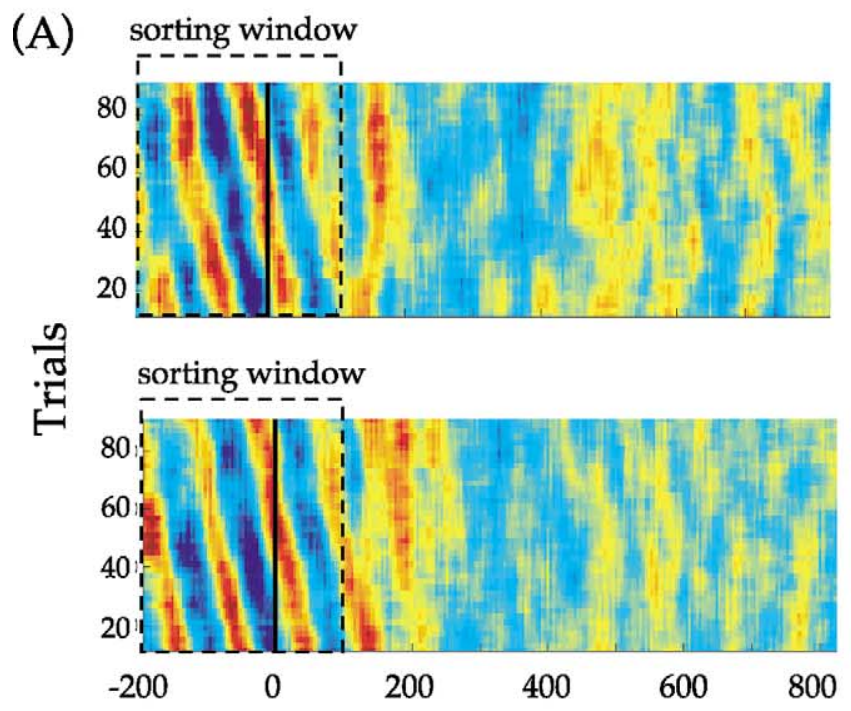

(B)

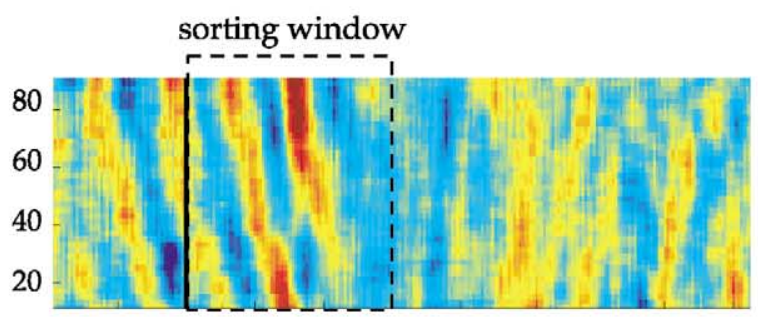

先

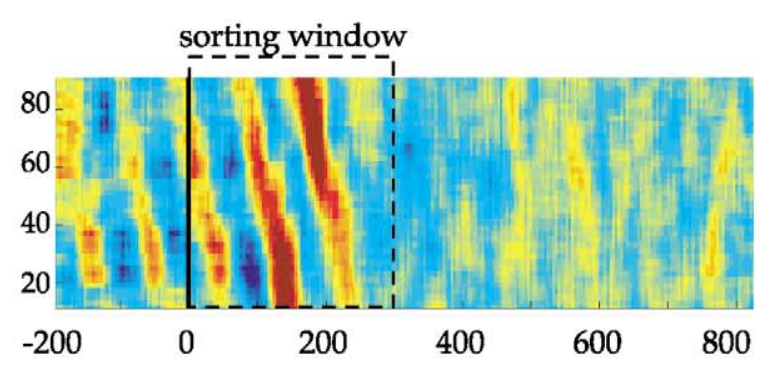

(C)
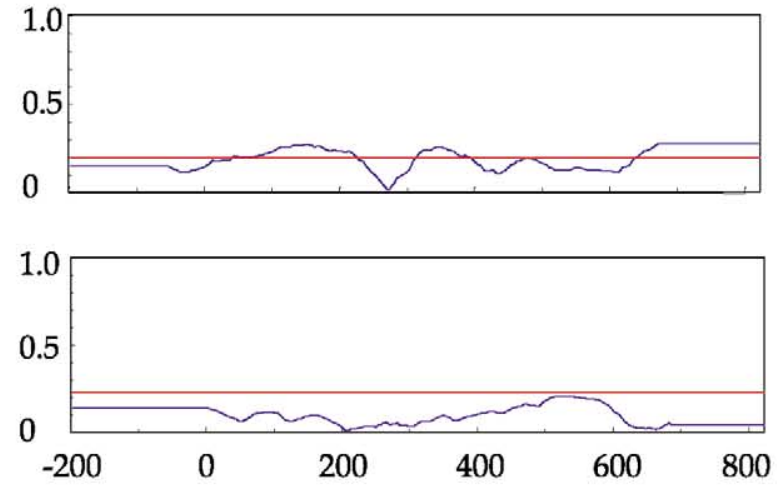

\section{Time (ms)}

Fig. 8. Relationship between alpha $(10 \mathrm{~Hz})$ phase of the activation waveforms $(n=100)$ of the IC representing parieto-occipital (PO) activity. Details are as reported in the legend to Fig. 7. 


\section{Effects of attentional modulation on beta and theta activity}

When visual attention was directed toward the stimulus, enhanced beta activity (13-20 Hz) adjacent to calcarine sulcus in the left (contralateral) hemisphere was evident between 200 and $400 \mathrm{~ms}$ poststimulus onset (Fig. 4C). Because the time course of the changes in alpha and beta activity within the calcarine cortex was markedly different, we assume that the two effects subserve different functions. While the sustained increase in alpha rhythm is probably related to the attentional status of the observer (Foxe et al., 1998; Worden et al., 2000), the delayed enhancement of beta activity may reflect changes in sensory processing (Martinez et al., 2001).

A reduction in theta activity $(5-6 \mathrm{~Hz})$ within the calcarine, occurring shortly after the onset of the stimulus and lasting for $400 \mathrm{~ms}$, was also evident in the contralateral hemipshere. This may relate to oscillatory activity within thalamo-cortical relay neurons (Lopes da Silva, 1991). If the thalamo-cortico-thalamic interaction plays an important role in attention (Crick, 1984; O'Connor et al., 2002; Raizada and Grossberg, 2003), enhanced alpha activity may mirror reduced theta activity.

\section{Acknowledgments}

We thank T. Tomita and S. Kajihara for their support with the MEG recordings. This study was supported by Special Coordination Funds for promoting Science and Technology from the Science and Technology Agency of the Japanese Government.

\section{References}

Anderson, S.J., 2002. Functional neuroimaging in amblyopia, in: Mosely, M., Fielder, A. (Eds.), Amblyopia: A Multidisciplinary Approach, Butterworth Heinmann, Oxford, pp. 43-67.

Anderson, S.J., Holliday, I.E., Harding, G.F., 1999. Assessment of cortical dysfunction in human strabismic amblyopia using magnetoencephalography (MEG). Vision Res. 39, 1723-1738.

Anderson, S.J., Holliday, I.E., Singh, K.D., Harding, G.F., 1996. Localization and functional analysis of human cortical area V5 using magneto-encephalography. Proc. R. Soc. Lond. B Biol. Sci. 263, 423-431.

Auger, F., Flandrin, P., Goncalves, P., Lemoine, O., 1996. Time-Frequency Toolbox: for Use with Matlab. Centre National de la Recherche Scientifique, France.

Barnes, G.R., Hess, R.F., Dumoulin, S.O., Achtman, R.L., Pike, G.B., 2001. The cortical deficit in humans with strabismic amblyopia. J. Physiol. 533, 281-297.

Brandt, M.E., 1997. Visual and auditory evoked phase resetting of the alpha EEG. Int. J. Psychophysiol. 26, 285-298.

Brefczynski, J.A., DeYoe, E.A., 1999. A physiological correlate of the "spotlight" of visual attention. Nat. Neurosci. 2, 370-374.

Callan, D.E., Callan, A.M., Kroos, C., Vatikiotis-Bateson, E., 2001. Multimodal contribution to speech perception revealed by independent component analysis: a single-sweep EEG case study. Brain Res. Cogn. Brain Res. 10, 349-353.
Chatila, M., Milleret, C., Buser, P., Rougeul, A., 1992. A 10 Hz "alphalike" rhythm in the visual cortex of the waking cat. Electroencephalogr. Clin. Neurophysiol. 83, 217-222.

Chawla, D., Rees, G., Friston, K.J., 1999. The physiological basis of attentional modulation in extrastriate visual areas. Nat. Neurosci. 2, 671-676.

Clark, V.P., Hillyard, S.A., 1996. Spatial selective attention affects early extrastriate but not striate components of the visual evoked potential. J. Cogn. Neurosci. 8, 387-402.

Crick, F., 1984. Function of the thalamic reticular complex: the searchlight hypothesis. Proc. Natl. Acad. Sci. USA 81, 4586-4590.

Engel, S., Zhang, X., Wandell, B., 1997. Colour tuning in human visual cortex measured with functional magnetic resonance imaging. Nature $388,68-71$.

Foxe, J.J., Simpson, G.V., Ahlfors, S.P., 1998. Parieto-occipital approximately $10 \mathrm{~Hz}$ activity reflects anticipatory state of visual attention mechanisms. NeuroReport 9, 3929-3933.

Fries, P., Reynolds, J.H., Rorie, A.E., Desimone, R., 2001. Modulation of oscillatory neuronal synchronization by selective visual attention. Science 291, 1560-1563.

Fylan, F., Holliday, I.E., Singh, K.D., Anderson, S.J., Harding, G.F., 1997. Magnetoencephalographic investigation of human cortical area V1 using color stimuli. NeuroImage 6, 47-57.

Gandhi, S.P., Heeger, D.J., Boynton, G.M., 1999. Spatial attention affects brain activity in human primary visual cortex. Proc. Natl. Acad. Sci. USA 96, 3314-3319.

Hamalainen, M., Hari, R., Ilmoniemi, R.J.e.a., Knuutila, J., Lounasmaa, O.V., 1993. Magnetoencephalography - theory, instrumentation and applications to non-invasive studies of the working human brain. Rev. Mod. Phys. 65, 413-497.

Heinze, H.J., Mangun, G.R., Burchert, W., Hinrichs, H., Scholz, M., Munte, T.F., Gos, A., Scherg, M., Johannes, S., Hundeshagen, H., et al., 1994. Combined spatial and temporal imaging of brain activity during visual selective attention in humans. Nature 372, 543-546.

Imada, T., Kawakatsu, M., Kotani, M., 1996. Attention-related enhancement of extrastriate neuromagnetic signals, in: Ogura, C., Koga, Y., Shimokochi, M. (Eds.), Recent Advances in Event-Related Brain Potential Research, Elsevier Science, New York, pp. 499-503.

Ito, M., Gilbert, C.D., 1999. Attention modulates contextual influences in the primary visual cortex of alert monkeys. Neuron 22, 593-604.

Jeffreys, D.A., Axford, J.G., 1972. Source locations of pattern-specific components of human visual evoked potentials. I. Component of striate cortical origin. Exp. Brain Res. 16, 1-21.

Jensen, O., Gelfand, J., Kounios, J., Lisman, J.E., 2002. Oscillations in the alpha band $(9-12 \mathrm{~Hz})$ increase with memory load during retention in a short-term memory task. Cereb. Cortex 12, 877-882.

Kajihara, S., Tomita, S., Kondo, Y., Arakawa, A., Okamura, S., Tomita, T., Yoshida, Y., Takanashi, Y., 2000. Moving mesh method for reconstructing some spread sources in the brain. Brain Topogr. 12, 283-292.

Kastner, S., Pinsk, M.A., De Weerd, P., Desimone, R., Ungerleider, L.G., 1999. Increased activity in human visual cortex during directed attention in the absence of visual stimulation. Neuron 22, 751-761.

Klimesch, W., 1999. EEG alpha and theta oscillations reflect cognitive and memory performance: a review and analysis. Brain Res. Brain Res. Rev. 29, 169-195.

Krauskopf, J., Williams, D.R., Heeley, D.W., 1982. Cardinal directions of color space. Vision Res. 22, 1123-1131.

Lee, D.K., Koch, C., Braun, J., 1997. Spatial vision thresholds in the near absence of attention. Vision Res. 37, 2409-2418.

Logothetis, N.K., Pauls, J., Augath, M., Trinath, T., Oeltermann, A., 2001. Neurophysiological investigation of the basis of the fMRI signal. Nature 412, 150-157.

Lopes da Silva, F., 1991. Neural mechanisms underlying brain waves: from neural membranes to networks. Electroencephalogr. Clin. Neurophysiol. 79, 81-93. 
Luck, S.J., Chelazzi, L., Hillyard, S.A., Desimone, R., 1997. Neural mechanisms of spatial selective attention in areas V1, V2, and V4 of macaque visual cortex. J. Neurophysiol. 77, 24-42.

Makeig, S., Humphries, C., Jung, T.P., Bell, T., McKeown, M., Dimitrov, A., Lee, T.W., Cardoso, J.F., 2001. MATLAB functions for Psychophysiological Data Analysis. CNL/Salk Institute.

Makeig, S., Jung, T.P., Bell, A.J., Ghahremani, D., Sejnowski, T.J., 1997. Blind separation of auditory event-related brain responses into independent components. Proc. Natl. Acad. Sci. USA 94, 10979-10984.

Makeig, S., Westerfield, M., Jung, T.P., Enghoff, S., Townsend, J., Courchesne, E., Sejnowski, T.J., 2002. Dynamic brain sources of visual evoked responses. Science 295, 690-694.

Mangun, G.R., Buonocore, M.H., Girelli, M., Jha, A.P., 1998. ERP and fMRI measures of visual spatial selective attention. Hum. Brain Mapp. 6, 383-389.

Martinez, A., Anllo-Vento, L., Sereno, M.I., Frank, L.R., Buxton, R.B., Dubowitz, D.J., Wong, E.C., Hinrichs, H., Heinze, H.J., Hillyard, S.A., 1999. Involvement of striate and extrastriate visual cortical areas in spatial attention. Nat. Neurosci. 2, 364-369.

Martinez, A., DiRusso, F., Anllo-Vento, L., Sereno, M.I., Buxton, R.B., Hillyard, S.A., 2001. Putting spatial attention on the map: timing and localization of stimulus selection processes in striate and extrastriate visual areas. Vision Res. 41, 1437-1457.

Mehta, A.D., Ulbert, I., Schroeder, C.E., 2000. Intermodal selective attention in monkeys. I: distribution and timing of effects across visual areas. Cereb. Cortex 10, 343-358.

Moran, J., Desimone, R., 1985. Selective attention gates visual processing in the extrastriate cortex. Science 229, 782-784.

Motter, B.C., 1993. Focal attention produces spatially selective processing in visual cortical areas V1, V2, and V4 in the presence of competing stimuli. J. Neurophysiol. 70, 909-919.

O'Connor, D.H., Fukui, M.M., Pinsk, M.A., Kastner, S., 2002. Attention modulates responses in the human lateral geniculate nucleus. Nat. Neurosci. 5, 1203-1209.

Pfurtscheller, G., 2001. Functional brain imaging based on ERD/ERS Vision Res. 41, 1257-1260.

Pfurtscheller, G., Lopes da Silva, F.H., 1999. Event-related EEG/MEG synchronization and desynchronization: basic principles. Clin. Neurophysiol. 110, 1842-1857.

Posner, M.I., Petersen, S.E., 1990. The attention system of the human brain. Annu. Rev. Neurosci. 13, 25-42.

Raizada, R.D., Grossberg, S., 2003. Towards a theory of the laminar architecture of cerebral cortex: computational clues from the visual system. Cereb. Cortex 13, 100-113.

Ray, W.J., Cole, H.W., 1985. EEG alpha activity reflects attentional demands, and beta activity reflects emotional and cognitive processes. Science 228, 750-752.
Ress, D., Backus, B.T., Heeger, D.J., 2000. Activity in primary visual cortex predicts performance in a visual detection task. Nat. Neurosci. 3 , 940-945.

Roelfsema, P.R., Lamme, V.A., Spekreijse, H., 1998. Object-based attention in the primary visual cortex of the macaque monkey. Nature 395 , 376-381

Singh, K.D., Barnes, G.R., Hillebrand, A., Forde, E.M., Williams, A.L., 2002. Task-related changes in cortical synchronization are spatially coincident with the hemodynamic response. NeuroImage 16, 103-114.

Smith, A.T., Singh, K.D., Greenlee, M.W., 2000. Attentional suppression of activity in the human visual cortex. NeuroReport 11, 271-277.

Somers, D.C., Dale, A.M., Seiffert, A.E., Tootell, R.B., 1999. Functional MRI reveals spatially specific attentional modulation in human primary visual cortex. Proc. Natl. Acad. Sci. USA 96, 1663-1668.

Steinmetz, P.N., Roy, A., Fitzgerald, P.J., Hsiao, S.S., Johnson, K.O., Niebur, E., 2000. Attention modulates synchronized neuronal firing in primate somatosensory cortex. Nature 404, 187-190.

Talairach, J., Tournoux, P., 1988. Co-planar Stereotaxic Atlas of the Human Brain: 3-Dimensional Proportional System: An Approach to Cerebral Imaging. Thieme, New York.

Tallon-Baudry, C., Bertrand, O., 1999. Oscillatory gamma activity in humans and its role in object representation. Trends Cogn. Sci. 3, $151-162$.

Tallon-Baudry, C., Bertrand, O., Delpuech, C., Pernier, J., 1996. Stimulus specificity of phase-locked and non-phase-locked $40 \mathrm{~Hz}$ visual responses in human. J. Neurosci. 16, 4240-4249.

Tootell, R.B., Reppas, J.B., Kwong, K.K., Malach, R., Born, R.T., Brady, T.J., Rosen, B.R., Belliveau, J.W., 1995. Functional analysis of human MT and related visual cortical areas using magnetic resonance imaging. J. Neurosci. 15, 3215-3230.

Treisman, A.M., Gelade, G., 1980. A feature-integration theory of attention. Cog. Psychol. 12, 97-136.

Vanni, S., Revonsuo, A., Hari, R., 1997. Modulation of the parietooccipital alpha rhythm during object detection. J. Neurosci. 17, 71417147.

Vidyasagar, T.R., 1998. Gating of neuronal responses in macaque primary visual cortex by an attentional spotlight. NeuroReport 9, 1947-1952.

von Stein, A., Chiang, C., Konig, P., 2000. Top-down processing mediated by interareal synchronization. Proc. Natl. Acad. Sci. USA 97, $14748-$ 14753.

Watanabe, T., Harner, A.M., Miyauchi, S., Sasaki, Y., Nielsen, M., Palomo, D., Mukai, I., 1998. Task-dependent influences of attention on the activation of human primary visual cortex. Proc. Natl. Acad. Sci. USA 95, 11489-11492.

Worden, M.S., Foxe, J.J., Wang, N., Simpson, G.V., 2000. Anticipatory biasing of visuospatial attention indexed by retinotopically specific alpha-band electroencephalography increases over occipital cortex. J. Neurosci. 20, RC63(61-66). 